\title{
The first approved treatment in children with multiple sclerosis slows brain atrophy
}

Arman Eshaghi ${ }^{1,2}, \mathrm{MD}, \mathrm{PhD}$

${ }^{1}$ Queen Square Multiple Sclerosis Centre, Department of Neuroinflammation, Queen Square Institute of Neurology, Faculty of Brain Sciences, University College London, London, UK WC1B5EH

${ }^{2}$ Centre for Medical Image Computing, Department of Computer Science, Faculty of Engineering Sciences, University College London, London, UK

\section{a.eshaghi@ucl.ac.uk}

Multiple sclerosis (MS) is a common disease in young adults but rare in children. More than a dozen treatments have been approved by the US Food and Drug Administration (FDA) for treating multiple sclerosis (MS) in adults and several of these treatments are prescribed in children. However, high-quality targeted evidence for children has been lacking. A recent phase 3 double-blind randomised controlled trial of fingolimod (PARADIGMS) vs interferon beta-1a was the first to show that fingolimod significantly reduced the relapse rate by $82 \%$ and appearance of new or enlarging lesions on T2-weighted magnetic resonance imaging (MRI) by $53 \%$. These benefits came at the cost of more serious adverse events in children with MS [1].

It is well known that similar to adults, loss of brain tissue (or brain atrophy) occurs in children with MS [2]. However, it was not known if brain atrophy could be slowed in children with MS, until now. As a follow-up study to PARADIGMS, in this issue, the paper by Arnold et al on $p$ XXX [ADD CITATION] reports the brain MRI results of this trial: fingolimod reduced the accrual of lesions on T2-weighted, gadolinium-enhancing, and new hypointense lesions on T1weighted MRI. Importantly, this paper shows that fingolimod reduced the rate of brain atrophy in children with MS up to $40 \%$ compared to the interferon beta-1a treatment (absolute difference of $0.32 \%$ per year). Therefore, similar to adults [3], children with MS benefit from 
the fingolimod treatment due to a direct anti-inflammatory effect, with a possible secondary effect on brain atrophy.

Although these results were expected and the sample size of children with age of less than 12 was limited in the trial, Arnold et al's MRI study contributes significantly to the field for several reasons. With a high-quality study design, it underlines the utility of brain atrophy as an outcome measure in children with MS, as we move forward to screen and test innovative treatments in phase 2 trials with reduced sample sizes. Although paediatric MS is a more inflammatory disease than the adult MS, the neurodegeneration-as measured by brain atrophy on MRI-is an important ongoing pathology, which can be slowed. These findings have important practical implications on the timing of treatments to slow the brain damage in children. Secondly, this study constructs a benchmark to test future therapies. Conducting double-blind trials in paediatric MS has been challenging partly due to ethical and treatmentblinding difficulties of injectable interferons with sham interventions for a rare disease. PARADIGMS paves the way to conduct more feasible multi-centre double-blind randomized controlled trials with fingolimod as an active comparator; perhaps even more so than the accelerated journey of oral treatments in adults after the approval of fingolimod [3]. As we improve in controlling the inflammatory manifestations of MS in children, the neurodegenerative aspect starts to become the focus of future research.

\section{Disclosures}

I have received funding for travel from the US National Multiple Sclerosis Society, speaker's honorarium (paid to my institution) from Biogen, and At The Limits educational programme.

\section{References}

1 Chitnis T, Arnold DL, Banwell B, et al. Trial of Fingolimod versus Interferon Beta-1a in Pediatric Multiple Sclerosis. N Engl J Med 2018;379:1017-27. doi:10.1056/NEJMoa1800149

2 De Meo E, Meani A, Moiola L, et al. Dynamic gray matter volume changes in pediatric multiple sclerosis: A 3.5 year MRI study. Neurology 2019;92:e1709-23. doi:10.1212/WNL.0000000000007267

3 Cohen JA, Barkhof F, Comi G, et al. Oral fingolimod or intramuscular interferon for relapsing multiple sclerosis. N Engl J Med 2010;362:402-15. doi:10.1056/NEJMoa0907839 
